\title{
A QUÍMICA NA REGIÃO NORTE
}

\author{
Alberto Cardoso Arruda
}

Departamento de Química - Centro de Ciências Exatas e Naturais - Universidade Federal do Pará - CP 8600 - 66057-970 - Belém - PA

\begin{abstract}
CHEMISTRY IN NORTH BRAZIL. A historical overview of CHEMISTRY SCIENCE from the beginning of the ESCOLA DE QUÍMICA INDUSTRIAL DO PARÁ in the northern region of Brazil is given. Reports were conducted on graduate and under graduate coursework within research and teaching institutions in each State of the Amazon region. Data collection included teaching, research and industrial activities.
\end{abstract}

Keywords: chemistry in Amazon region; teaching; research; industry.

Somos uma região de dimensões continentais. Nos milhões de quilômetros quadrados de que se constitui nosso território, mais exatamente $3.869 .637,9 \mathrm{Km}^{2}$, correspondentes a $45 \%$ da superfície do Brasil e mais de duas vezes a área da segunda maior região brasileira, riquezas múltiplas se irradiam ${ }^{1}$. Além da opulência de nossos recursos naturais e da grandiosidade indiscutível de cada pedaço de nossa natureza, a Amazônia é uma terra onde o povo cria cultura de matizes variados, e todos de inegável beleza. Nossas lendas, músicas, e arte caminham nos meandros do lirismo, renovam-se na vitalidade de nossa juventude e revelam a sabedoria de nossos velhos. Tão valioso este patrimônio que, quaisquer que sejam os brasileiros, havemos de manter a lucidez necessária à sua preservação ilimitada.

A região Norte é considerada um grande vazio demográfico quando comparada a outras regiões brasileiras. O advento da rodovia Belém-Brasília tirou a Amazônia do isolamento terrestre e através dela entraram também os conceitos de modernização para o desenvolvimento da região nos anos 60 , hoje claramente avaliados como equivocados ${ }^{2}$.

As mudanças foram sendo implantadas sem que, a princípio, a sociedade se apercebesse disso. Inicialmente veio a operação desmonte com a retirada das estradas de ferro, a construção da Transamazônica e o avanço do desmatamento. Florestas densas foram substituídas por pastagens sem futuro e com elas foram perdidas plantas e animais que sequer haviam sido inventariados. Em seguida vieram os megaprojetos de colonização, como o Programa Pólo Noroeste em Rondônia, onde centenas de milhares de migrantes desembarcaram e passaram a ocupar as margens das novas estradas, para desenvolver uma agricultura em clima e solo desconhecidos. Passamos então a assistir o desenvolvimento industrial, surgiram a Zona Franca de Manaus e alguns pólos metalúrgicos como o de Carajás e o de Barcarena no Pará. A exploração de madeira foi incentivada e expandida. Tal desenvolvimento passou a exigir da região maior geração de energia elétrica. Surgiram as grandes hidrelétricas como a de Tucuruí que mesmo levando energia para o Nordeste brasileiro, ainda não alimenta a maioria dos municípios do Pará.

Obviamente a busca de um melhor destino para a Amazônia ainda parece distante. Nestes últimos anos do século $\mathrm{XX}$ temos vivido grandes preocupações: será que a imensa floresta sobreviverá às recentes depredações? Serão as populações indígenas arrasadas e dizimadas cultural e biologicamente? Terá a biotecnologia do futuro acesso às riquezas genéticas da Amazônia? Aprenderemos a usar os recursos vegetais que hoje consideramos não utilizáveis? Criaremos a tecnologia necessária para o correto uso do solo? Poderão a medicina, a

E-Mail: arruda@marajo.ufpa.br farmacologia e a fitoquímica descobrir na floresta novas substâncias químicas capazes de produzir a cura de doenças classificadas como incuráveis?

A Amazônia não é um grande vazio demográfico ou cultural. Suas populações conhecem as características de cada localidade $^{3}$. Para preservar e, ao mesmo tempo, desenvolver a região é necessário, a partir de seus habitantes, aprender sobre a complexidade de seu mundo.

Dentro deste contexto, cabe também a nós profissionais da química, que desenvolvemos atividades na região Norte, uma considerável parcela de responsabilidade no processo do desenvolvimento regional. É grande o desafio de entendermos as particularidades da Amazônia, muitas das vezes tendo que deixar de lado os conhecimentos e as tecnologias gerados e adquiridos em outros locais, freqüentemente inapropriados às características regionais, passando então a produzir novos conhecimentos e tecnologias adaptados ao dia-a-dia amazônico.

Desde a origem da Química na Amazônia, caracterizada pelas atividades desenvolvidas na Escola de Química Industrial do Pará, fundada em 1920, as atividades voltadas para o conhecimento e a exploração racional dos recursos naturais da região têm sido a principal motivação para a pesquisa e a formação de recursos humanos.

Em 1956, a Escola de Química Industrial do Pará recebeu a denominação de Escola Superior de Química do Pará, sendo responsável pela formação de centenas de profissionais de Química que, ainda hoje, exercem atividades na indústria e em instituições de ensino e pesquisa em todo o país, especialmente nos estados do Pará, Amazonas e Maranhão.

Absorvida pela Universidade Federal do Pará em 1963 e extinta em 1970, quando da Reforma Universitária, esta Escola deu origem a dois departamentos didáticos científicos, o Departamento de Química do Centro de Ciências Exatas e Naturais e o Departamento de Engenharia Química do Centro Tecnológico.

Coletar as informações que aqui estão reunidas não foi tarefa das mais fáceis, principalmente levando-se em consideração as grandes distâncias. Manaus, por exemplo, está praticamente tão distante de Belém quanto Brasília (aprox. $2.000 \mathrm{Km}$ ), a região possui três fusos-horários (8:00 horas em Rio Branco correspondem a 9:00 horas em Manaus e 10:00 horas em Belém), voa-se mais de uma hora de jato entre duas cidades em um mesmo estado. Tais características somadas a outros fatores têm levado, muitas vezes, os grupos que desenvolvem ensino e pesquisa na área da química a terem mais facilidade de interagir com grupos de outras regiões que entre si.

Os profissionais de química da região Norte estão agrupados em dois Conselhos Regionais, o CRQ-6 $6^{\text {a }}$ Região e o CRQ-14aㅡ Região (Quadro 1). 
Na região Norte, a atividade industrial voltada para a área da química ainda não é tão expressiva. Um crescimento considerável vem sendo registrado dentro do ensino e da pesquisa, centralizado principalmente nas universidades federais e em alguns institutos de pesquisa (Quadros 2 a 14). A carência de pessoal com alta qualificação tem levado tais grupos a programas agressivos de formação de recursos humanos, o que é confirmado pelo crescente número de doutores e mestres e pelo grande número de profissionais que se encontram em treinamento. Já dispondo de cursos de pós-graduação de bom nível, a região começa finalmente, a formar seus próprios profissionais, moldados à sua realidade e prontos a difundir seus conhecimentos.

Outra característica importante a ser citada, é a centraliza- ção de todo arsenal de ensino e pesquisa nas capitais. Programas de interiorização das universidades vem sendo implantados buscando levar para fora das capitais a oportunidade para a formação de recursos humanos. Um dos programas de referência é o da Universidade Federal do Pará a qual está atuando em oito cidades do interior do estado.

\section{A QUímica nO ESTADO DO PARÁ}

Os docentes que atuam no Curso de Pós-Graduação em Química pertencem ao quadro do Departamento de Química, e aqueles que atuam no Curso de Pós-Graduação em Engenharia Química pertencem ao quadro do Departamento de Engenharia Química.

Quadro 1. Profissionais de Química da Região Norte.

\begin{tabular}{cccc}
\hline Conselho & Estados & $\begin{array}{c}\text { No de } \\
\text { profissionais }\end{array}$ & $\begin{array}{c}\text { Sede } \\
\text { Registr. }\end{array}$ \\
\hline $6^{-a}$ Região & Pará e Amapá & 702 & Belém \\
$14^{\text {a }}$ Região & $\begin{array}{c}\text { Amazonas, Acre, } \\
\text { Rondônia e Roraima }\end{array}$ & 448 & Manaus \\
\hline
\end{tabular}

* não há dados sobre o estado do Tocantins.

Quadro 2. Universidade Federal do Pará (ensino de graduação).

\begin{tabular}{|c|c|c|c|c|c|c|c|c|}
\hline \multirow[t]{2}{*}{ Centro } & \multirow[t]{2}{*}{ Departamento } & \multirow[t]{2}{*}{ Cursos } & \multirow[b]{2}{*}{ PHD } & \multicolumn{5}{|c|}{ Corpo Docente (Qualificação) } \\
\hline & & & & DO & $\mathrm{ME}$ & $\mathrm{ES}$ & GR & TR \\
\hline $\begin{array}{l}\text { Ciências } \\
\text { Exatas e } \\
\text { Naturais }\end{array}$ & Química & $\begin{array}{l}\text { Licenciatura } \\
\text { Bacharelado }\end{array}$ & 01 & 18 & 08 & 05 & 12 & 05 \\
\hline Tecnológico & $\begin{array}{l}\text { Engenharia } \\
\text { Química }\end{array}$ & $\begin{array}{l}\text { Quim. Industr. } \\
\text { Eng. Química }\end{array}$ & 00 & 05 & 09 & 01 & 00 & 12 \\
\hline
\end{tabular}

PHD (pós-doutor), DO (doutor), ME (mestre), ES (especialista), GR (graduado) e TR (em treinamento)

Quadro 3. Universidade Federal do Pará (pesquisa).

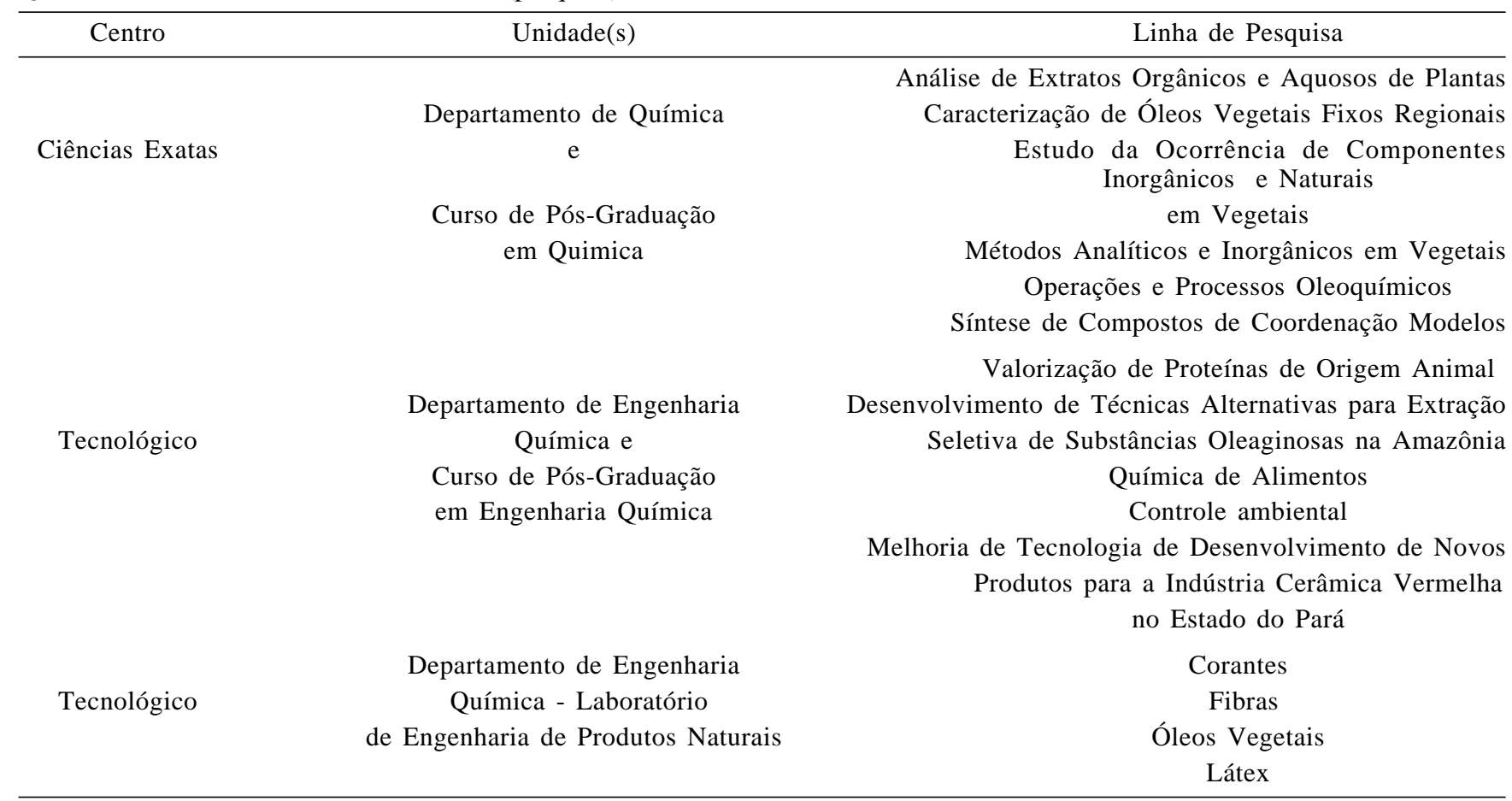


Quadro 4. Universidade Federal do Pará (ensino de pós-graduação).

\begin{tabular}{|c|c|c|c|c|c|c|c|c|c|}
\hline \multirow[t]{2}{*}{ Centro } & \multirow[t]{2}{*}{ Curso } & \multirow[t]{2}{*}{ Áreas } & \multirow[t]{2}{*}{ Nível } & \multicolumn{3}{|c|}{ Docentes } & \multicolumn{2}{|c|}{ Alunos } & \multirow{2}{*}{$\begin{array}{l}\text { Dissertações } \\
\text { Defendidas }\end{array}$} \\
\hline & & & & PHD & DO & ME & Do & $\mathrm{Me}$ & \\
\hline $\begin{array}{c}\text { Ciências Exatas } \\
\text { e Naturais }\end{array}$ & $\begin{array}{c}\text { Pós-Graduação } \\
\text { em Química }\end{array}$ & $\begin{array}{l}\text { Q. Orgânica } \\
\text { e Físico-Quim. } \\
\text { Inorgânica }\end{array}$ & $\begin{array}{c}\text { Mestado } \\
\mathrm{e} \\
\text { Doutorado }\end{array}$ & 01 & 15 & 01 & 04 & 30 & 20 \\
\hline Tecnológico & $\begin{array}{l}\text { Pós-Grad. em } \\
\text { Eng. Química }\end{array}$ & & Mestrado & 00 & 06 & 04 & 00 & 20 & 10 \\
\hline
\end{tabular}

PHD (Docente com pós-doutorado), DO (doutor), ME (mestre), Do (doutorando), Me (mestrando).

Quadro 5. Outras instituições de ensino e pesquisa no Pará.

\begin{tabular}{|c|c|c|c|c|c|c|c|c|c|}
\hline \multirow[t]{2}{*}{ Instituição } & \multirow{2}{*}{$\begin{array}{l}\text { Área de } \\
\text { Atuação }\end{array}$} & \multirow{2}{*}{$\begin{array}{l}\text { Departamento/ } \\
\text { Laboratório }\end{array}$} & \multicolumn{5}{|c|}{ Docentes/Pesquisadores } & \multirow{2}{*}{$\begin{array}{c}\text { Áreas de } \\
\text { conhecimento }\end{array}$} & \multirow{2}{*}{$\begin{array}{l}\text { Linhas de } \\
\text { Pesquisa }\end{array}$} \\
\hline & & & DO & ME & $\mathrm{ES}$ & GR & $\mathrm{TC}$ & & \\
\hline $\begin{array}{c}\text { Faculdade } \\
\text { de Ciências } \\
\text { Agrárias do Pará }\end{array}$ & $\begin{array}{c}\text { Ensino } \\
\text { de graduação }\end{array}$ & $\begin{array}{l}\text { Departamento } \\
\text { de Química } \\
\text { e Tecnologia }\end{array}$ & 02 & 02 & 01 & 00 & 01 & $\begin{array}{c}\text { Medicina } \\
\text { Veterinária } \\
\text { Agronomia } \\
\text { Eng. Florestal }\end{array}$ & $\begin{array}{c}\text { Cultura de } \\
\text { Tecidos, Ciência } \\
\text { e Tecnologia } \\
\text { de Alimentos }\end{array}$ \\
\hline $\begin{array}{c}\text { Faculdade } \\
\text { de Ciências } \\
\text { Agrárias do Pará }\end{array}$ & $\begin{array}{l}\text { Pós-Graduação } \\
\text { (especialização) }\end{array}$ & $\begin{array}{l}\text { Departamento } \\
\text { de Química } \\
\text { e Tecnologia }\end{array}$ & 02 & 02 & 01 & 00 & 01 & $\begin{array}{c}\text { Tecnologia } \\
\text { do Pescado } \\
\text { Processamento de } \\
\text { Frutas Tropicais } \\
\text { e Hortaliças }\end{array}$ & \\
\hline $\begin{array}{c}\text { Museu Paraense } \\
\text { Emílio Goeldi/CNPq }\end{array}$ & Pesquisa & $\begin{array}{l}\text { Departamento } \\
\text { de Botânica }\end{array}$ & 02 & 02 & 00 & 00 & 00 & & $\begin{array}{c}\text { Óleos Essenciais } \\
\text { Amazônicos } \\
\text { Aromas de Frutas } \\
\text { da Amazônia }\end{array}$ \\
\hline EMBRAPA-PA & Pesquisa & $\begin{array}{l}\text { Laboratório } \\
\text { de Agroindústria }\end{array}$ & 00 & 02 & 00 & 03 & 00 & & $\begin{array}{c}\text { Plantas Medicinais } \\
\text { Alimentos }\end{array}$ \\
\hline
\end{tabular}

DO (doutor), ME (mestre), ES (especialista), GR (graduado), TC (técnico).

\section{A QUímica no ESTAdo dO AMAZONAS}

Quadro 6. Fundação Universidade Federal do Amazonas (ensino de graduação).

\begin{tabular}{cccccc}
\hline Instituto & Departamento & Cursos & \multicolumn{3}{c}{ Corpo Docente (Qualificação) } \\
GR & DO & ME & ES \\
\hline Ciências & Química & Licenciatura & & & \\
Exatas & & Bacharelado & 03 & 12 & 01 \\
\hline
\end{tabular}

DO (doutor), ME (mestre), ES (especialista), GR (graduado) e TR (em treinamento).

Quadro 7. Fundação Universidade Federal do Amazonas (pesquisa).

\begin{tabular}{ccc}
\hline Instituto & Unidades & Linhas de Pesquisa \\
\hline & & Separação e Identificação de Componentes \\
Ciências & Químicos de Plantas Amazônicas \\
Exatas & de Química e & Síntese de Aldiminas Alifáticas \\
& Pós-graduação & Óleos Essenciais \\
& em Química de & Produtos \\
& Naturais & Determinação de Componentes Metálicos \\
& & em Plantas Amazônicas \\
& Ação Anti-ofídica de Produtos \\
\end{tabular}

Quadro 8. Fundação Universidade Federal do Amazonas (ensino de pós-graduação).

\begin{tabular}{|c|c|c|c|c|c|c|}
\hline Instituto & Unidade & Área & Nível & $\begin{array}{l}\text { Doc. } \\
\text { (DO) }\end{array}$ & $\begin{array}{l}\text { Disc } \\
(\mathrm{Me})\end{array}$ & $\begin{array}{c}\text { Dissertações } \\
\text { Defendidas }\end{array}$ \\
\hline $\begin{array}{l}\text { Ciências } \\
\text { Exatas }\end{array}$ & $\begin{array}{c}\text { Pós-graduação } \\
\text { em Química } \\
\text { de Produtos } \\
\text { Naturais }\end{array}$ & $\begin{array}{c}\text { Química de } \\
\text { Produtos Naturais }\end{array}$ & $\mathrm{ME}$ & $\begin{array}{l}03(\mathrm{e}) \\
04(\mathrm{p})\end{array}$ & 19 & 15 \\
\hline
\end{tabular}

(DO) docentes doutores, (e) efetivos, (p) participantes de outras IES, (Me) discentes mestrandos. 
Quadro 9. Instituto Nacional de Pesquisas da Amazônia (INPA) - pessoal.

\begin{tabular}{cccc}
\hline Setores & \multicolumn{3}{c}{ Pessoal/Qualificação } \\
& Doutores & Mestres & Graduados \\
\hline $\begin{array}{c}\text { Centro de Pesquisas } \\
\text { de Produtos Naturais } \\
\text { e }\end{array}$ & 02 & 12 & 07 \\
$\begin{array}{c}\text { Centro de Pesquisas } \\
\text { de Produtos Florestais }\end{array}$ & & & \\
\hline
\end{tabular}

\section{A QUÍMICA NO ESTADO DE RORAIMA}

Quadro 10. Instituto Nacional de Pesquisas da Amazônia (INPA) - linhas de pesquisa.

\begin{tabular}{cc}
\hline Setor & Linha de Pesquisa \\
\hline Centro de Pesquisas & Oleaginosas da Amazônia \\
de Produtos Naturais & Plantas Medicinais \\
& Óleos de Peixes \\
Interações Planta-Inseto & Ecologia Química \\
Centro de Pesquisas & Polímeros \\
de Produtos Florestais & Química da Madeira \\
& Microbiologia \\
\hline
\end{tabular}

Quadro 11. Universidade Federal de Roraima (ensino de graduação).

\begin{tabular}{cccccc}
\hline Centro & Departamento & Cursos & \multicolumn{3}{c}{ Corpo Docente (Qualificação) } \\
DR & PHD & DE & TR \\
\hline $\begin{array}{l}\text { Ciências e } \\
\text { Tecnologia }\end{array}$ & Química & Licenciatura & & & \\
Bacharelado & 01 & 01 & 04 & 02 \\
\hline
\end{tabular}

PHD (pós-doutor), DO (doutor), ME (mestre), GR (graduado) e TR (em treinamento).

Quadro 12. Universidade Federal de Roraima (pesquisa).

\begin{tabular}{ccc}
\hline Instituto & Unidade & Linhas de Pesquisa \\
\hline Ciências e & & Química de Produtos Naturais \\
Tecnologia & Departamento & Síntese Orgânica e de Corantes \\
& de Química & Química Teórica \\
Engenharia de Materiais
\end{tabular}

\section{A QUímica NO ESTADO DO ACRE}

Quadro 13. Universidade Federal do Acre (ensino de graduação).

\begin{tabular}{|c|c|c|c|c|c|}
\hline \multirow[t]{2}{*}{ Centro } & \multirow[t]{2}{*}{ Departamento } & \multirow[t]{2}{*}{ Cursos } & \multicolumn{3}{|c|}{ Docentes/Qualificação } \\
\hline & & & $\mathrm{ME}$ & GR & TR \\
\hline NI & $\begin{array}{l}\text { Ciências da } \\
\text { Natureza }\end{array}$ & $\begin{array}{c}\text { Habilitação em } \\
\text { Química }\end{array}$ & 02 & 01 & 03 \\
\hline
\end{tabular}

ME (mestres), GR (graduados), TR (em treinamento), NI (não informado).

Quadro 14. Universidade Federal do Acre (pesquisa).

\begin{tabular}{cc}
\hline Unidade & Linhas de Pesquisa \\
\hline Departamento de & Plantas Medicinais \\
Ciências da Natureza & Óleos Vegetais \\
\hline
\end{tabular}

\section{SITUAÇÃO DOS DEMAIS ESTADOS}

\section{Amapá}

A Universidade Federal do Amapá não possui um Departamento de Química, apenas alguns profissionais que atuam no Curso de Enfermagem. Não foi informado o número nem a qualificação destes profissionais. Não constam linhas de pesquisa ligadas especificamente à Química.

\section{Rondônia e Tocantins}

Não houve resposta às tentativas de obter informações destes estados.

Outras instituições de ensino superior particulares também abrigam profissionais de química na região, porém ainda não desenvolvem atividades voltadas diretamente para alguma área da química.
Fica patente, ao analisarmos os dados contidos nas tabelas, que as atividades de pesquisa, até então desenvolvidas na região, estão centralizadas no estudo dos recursos oriundos da fantástica biodiversidade amazônica. Sabe-se de estudos recentes $^{4}$, que a diversidade em espécies vegetais diminui com a distância do equador, o que concede à Amazônia uma alta diversidade em espécies.

Os grupos de pesquisa ainda estão centralizados nas duas maiores capitais da região, porém importantes nucleações começam a surgir em outras áreas como em Boa Vista e Rio Branco.

Entender quimicamente a Amazônia é antes de tudo uma declaração de amor a esta região. Um amor equatorial, tórrido, verdadeiro, cheio de dificuldades, muitas vezes incompreendido por nossos irmãos de outras regiões que, em certos momentos olham a Amazônia como um depósito onde é só chegar e levar o que for necessário, deixando-a com feridas irrecuperáveis. A fase do extrativismo já passou, toda ação na região deverá ter mão dupla. Muito temos a aprender aqui se tivermos a humildade de ceder aos ensinamentos de quem vive a Amazônia na Amazônia.

\section{AGRADECIMENTOS}

Meus mais sinceros agradecimentos aos profissionais que colaboraram com este artigo, respondendo as questões formuladas e 


\begin{tabular}{ccc}
\hline Adolfo Henrique Müller & DQ e CPGQPN - CCEN - UFPA & $(091) 2111363$ \\
Ayssor Paulo Mourão & DQ e PGPN - ICE - FUA & $(092) 6442006$ \\
Cláudio Roberto Orofino Pinto & DQ - CT - UFPA & $(092) 2111291$ \\
Délcio Dias Marques & DCN - UFAC & $(068) 2292244$ \\
Ester Levi & INPA - CPPN & $(092) 6433176$ \\
Irineide de Almeida Cruz & INPA - CPPF & $(092) 6423300$ \\
José Carlos A. Cardoso Filho & PGEQ-CT-UFPA & $(091) 2111908$ \\
José Luiz Moraes & DQT - Fac. Ciênc. Agrar. do Pará & $(091) 2105110$ \\
Lênio José G. de Farias & LEPRON-DEQ-CT-UFPA & $(091) 2111694$ \\
M. das Graças Bichara Zoghbi & Museu Paraense Emílio Goeldi & zoghbi@museu-goeldi.br \\
Maria Lúcia Taveira & DQ - CCT - UFRR & $(095) 6231581$ \\
Sérgio Bringel & CRQ-14á Região & $(092) 2338896$ \\
Sérgio de Melo Alves & EMBRAPA-PA & $(091) 2662311$ \\
Waterloo Napoleão de Lima & CRQ-6- Região & $(091) 2111365$ \\
\hline
\end{tabular}

enviando os dados pertinentes para sua elaboração. No sentido de facilitar o contato entre o leitor e estes profissionais, caso haja interesse de obter mais informações, relacionei-os no quadro acima.

\section{REFERÊNCIAS}

1. Bezerra, A.M. e Paula, A. M. T.; Lendas e Mitos da Amazônia, Delegacia do Ministério da Educação e Cultura do
Estado do Pará, Rio de Janeiro, 1985.

2. D’Incao, M. A. e Silveira, I. M.; Amazônia e a Crise da Modernização, Museu Paraense Emílio Goeldi, Belém, 1994.

3. Morán, E. F., A Ecologia Humana das Populações da Amazônia, Editora Vozes, Petrópolis, 1990.

4. Gottlieb, O. R., Kaplan, M. A. e Borin, M. R. de M. B.; Biodiversidade um Enfoque Químico-Biológico, Editora UFRJ, Rio de Janeiro, 1996. 